Patricia Carvalho de Jesus ${ }^{1}$

Maria Inês Couto de Oliveira ${ }^{1}$

José Rodrigo de Moraes ${ }^{2}$

\title{
Capacitação de profissionais de saúde em aleitamento materno e sua associação com conhecimentos, habilidades e práticas
}

\author{
Training of health professionals in breastfeeding \\ and its association with knowledge, skills and practices
}

${ }^{1}$ Programa de PósGraduação em Saúde Coletiva, Instituto de Saúde Coletiva, Universidade Federal Fluminense, Rua Marquês do Paraná 303/4 andar, Prédio Anexo, Centro. 24030-210. Niterói RJ Brasil.pcj.nutricao@ gmail.com

${ }^{2}$ Departamento de Estatística, UFF. Niterói RJ Brasil.
Abstract A cross-sectional study was conducted in the 15 hospitals with over 1000 deliveries/year in Rio de Janeiro, Brazil, to verify the association between training of health professionals in breastfeeding and professional knowledge, skills and practices. Interviews were staged with 215 health professionals, $48.4 \%$ working in Baby-Friendly Hospitals, by means of a questionnaire adapted from the revalidation instrument of the initiative. The three dichotomized outcomes were subjected to bivariate and multivariate analysis. Adjusted prevalence ratios were obtained by the Poisson regression model: $48.1 \%$ of the professionals had adequate knowledge, 58.9\% adequate skills and $74.9 \%$ reported adequate practice. Theoretical and practical training $\geq 18$ hours considered adequate (by $65.6 \%$ of the professionals) showed a significant association with professional knowledge $(a P R=1.575)$, skills $(a P R=1.530)$ and practices $(a P R=1.312)$. Less working experience was associated with less knowledge ( $a P R=$ $0.723)$, but with better practices $(a P R=1.183)$. Nursing staff reported better practices than physicians $(a P R=0.808)$ and other categories $(a P R=$ 0.658). The study concludes that training contributes to improved breastfeeding knowledge, skills and practices that are essential for maternal and child care.

Key words Training, Breastfeeding, Knowledge, Professional practice, Baby-friendly hospital initiative
Resumo Para verificar a associação entre capacitação em aleitamento materno e conhecimentos, habilidades e práticas profissionais, foi conduzido estudo transversal nos 15 hospitais com mais de 1000 partos/ano do município do Rio de Janeiro. Foram entrevistados 215 profissionais, sendo 48,4\% em Hospitais Amigos da Criança, por adaptação de questionário de reavaliação desta iniciativa. Os três desfechos, dicotomizados, foram utilizados em análises bivariadas e multivariadas, sendo obtidas razões de prevalência ajustadas por modelo de regressão de Poisson. Dos profissionais, 48,1\% tinham conhecimentos; $58,9 \%$ habilidades e $74,9 \%$ práticas adequadas. A capacitação teórico-prática $\geq 18$ horas, considerada adequada, presente em $65,6 \%$ dos profissionais, mostrou associação significativa com conhecimentos ( $R P a$ $=1,575)$, habilidades $(R P a=1,530)$ e práticas $(R P a=1,312)$. Profissionais com menor tempo de trabalho apresentaram menos conhecimentos $(R P a=0,723)$, mas relataram melhores práticas $(R P a=1,183)$. A enfermagem relatou melhores práticas em relação aos médicos $(R P a=0,808) e$ a outras categorias $(R P a=0,658)$. Conclui-se que a capacitação contribui para o aprimoramento de conhecimentos, habilidades e práticas em aleitamento materno, fundamentais à assistência materno-infantil.

Palavras-chave Capacitação, Aleitamento materno, Conhecimento, Prática profissional, Iniciativa Hospital Amigo da Criança 


\section{Introdução}

O aleitamento materno exclusivo por seis meses e complementado por outros alimentos até os 2 anos ou mais são preconizados pela Organização Mundial de Saúde $(\mathrm{OMS})^{1}$, pela sua importância na redução da morbimortalidade infantil por enfermidades comuns da infância, como a diarreia $^{2,3}$ e a pneumonia ${ }^{4}$.

A falta de apoio dos serviços de saúde, problemas de saúde do bebê, condições biológicas e psicológicas da mulher e o retorno ao trabalho são fatores que podem dificultar a prática de amamentação e levar à sua interrupção ${ }^{5}$.

A Iniciativa Hospital Amigo da Criança (IHAC) foi lançada pela OMS e pelo Fundo das Nações Unidas para a Infância (UNICEF) em 1991 objetivando a sensibilização, a capacitação e a mobilização dos profissionais de saúde das maternidades e hospitais para a instituição de normas, rotinas e condutas favoráveis à prática da amamentação, sintetizadas em "Dez Passos para o Sucesso do Aleitamento Materno"6. A IHAC tem sido responsável por mudanças expressivas na prevalência da amamentação e na duração do aleitamento materno exclusivo nos primeiros meses de vida ${ }^{3,6,7,8}$. Os profissionais de saúde são fundamentais na promoção e apoio ao aleitamento materno, e necessitam ter conhecimentos sobre o manejo da amamentação, bem como habilidades para auxiliarem as mães, por isso o curso da IHAC é teórico-prático9.

O objetivo deste estudo foi analisar a associação entre a capacitação em aleitamento materno e os conhecimentos, as habilidades e as práticas de profissionais de saúde que atuam na assistência a gestantes, mães e bebês em hospitais.

\section{Métodos}

Trata-se de estudo transversal, conduzido no segundo semestre de 2009 nos quinze hospitais com mais de 1000 partos/ano no município do Rio de Janeiro, que concentravam 94\% dos partos ocorridos no Sistema Único de Saúde (SUS), sendo sete hospitais amigos da criança e oito não credenciados na iniciativa ${ }^{10}$. A população de estudo foi composta por profissionais de saúde lotados nestes hospitais. Foi utilizado um plano amostral estratificado, sendo cada estrato uma unidade hospitalar, e cada profissional de saúde uma unidade elementar. Os profissionais de saúde foram selecionados utilizando uma amostragem aleatória simples, sem reposição, com base em cadastro fornecido pela direção de cada hospital. Foi adotada a alocação proporcional como método de alocação da amostra, considerando margem de erro máxima prévia de 5,0\%. Como houve cerca de $2 \%$ de perdas, esta margem de erro foi recalculada a posteriori, elevando-se para $6,8 \%{ }^{11}$. A amostra de profissionais de saúde definida para cada hospital abrangeu profissionais de diferentes categorias profissionais que atuavam há pelo menos seis meses nos setores de pré-natal, parto, alojamento conjunto e unidade neonatal informado por cada hospital (cerca de $4 \%$ do quantitativo de profissionais que atuavam nestes setores em cada hospital).

Foram utilizados questionários estruturados, adaptados do questionário de reavaliação da IHAC $^{12}$ para entrevista aos profissionais de saúde quanto aos conhecimentos, habilidades e práticas relativos aos Dez Passos para o Sucesso do Aleitamento Materno9, sendo acrescidas perguntas sobre características dos profissionais de saúde.

Este estudo teve aprovação do Comitê de Ética em Pesquisa da Secretaria Municipal de Saúde do Rio de Janeiro. Os dados foram colhidos mediante assinatura de Termo de Consentimento Livre e Esclarecido, contendo informações sobre a pesquisa, a não obrigatoriedade da participação no estudo, a identificação dos pesquisadores e instituições envolvidas, e a garantia da confidencialidade das informações prestadas e de sua utilização apenas de forma agregada.

Os questionários foram aplicados por três enfermeiras credenciadas pelo Ministério da Saúde como avaliadoras da IHAC, sob a supervisão da coordenadora da pesquisa, também avaliadora da IHAC. As perguntas eram realizadas sem serem enunciadas as possibilidades de resposta, e as entrevistadoras classificavam as respostas segundo as opções contidas no questionário.

Os questionários aplicados foram revistos e digitados, sendo criado um banco de dados pelo programa Epi-Info 2000. Os dados foram analisados através do programa estatístico SPSS 17.0.

A capacitação foi considerada adequada quando o profissional havia cursado um treinamento teórico-prático com carga horária mínima de 18 horas. Apesar da Organização Mundial de Saúde e do Ministério da Saúde terem passado a recomendar cursos de capacitação na IHAC de 20 horas a partir de $2008-2009^{13}$, incorporando ao curso de 18 horas um conteúdo relativo às habilidades de aconselhamento em amamentação, esta carga horária não foi exigida na presente avaliação, por tratar-se de recomendação recente, ainda não incorporada ao treinamento de vários 
profissionais de saúde capacitados previamente.

Inicialmente, foi realizada análise descritiva da amostra para caracterização da população do estudo. A capacitação adequada foi considerada a variável de exposição principal. $\mathrm{Na}$ análise foram consideradas também as variáveis de exposição: 1. credenciamento do local de trabalho na Iniciativa Hospital Amigo da Criança; 2. sexo do profissional; 3. tempo de trabalho, em duas categorias: de 6 meses a 9 anos, 10 anos ou mais; 4. profissão, em três categorias: médica, enfermagem e outras.

Os desfechos foram o conhecimento, as habilidades e as práticas dos profissionais de saúde. Todos os profissionais de saúde foram questionados sobre conhecimentos em aleitamento materno; suas habilidades, sendo solicitada demonstração de como seria uma pega/posição e ordenha manual da mama adequados; e sua prática em relação à orientação ou demonstração às mães dos tópicos "pega e posição" e "ordenha manual da mama”.

Cada desfecho foi aferido mediante uma série de perguntas. Na avaliação do conhecimento dos profissionais de saúde, este foi considerado adequado quando foram obtidas respostas corretas a quatro perguntas": 1. "Você considera que a oferta de qualquer alimento antes da primeira mamada interfere na amamentação?”, sendo a resposta afirmativa considerada correta; 2. "Qual a principal causa de dor no mamilo?", sendo a resposta "má pega" considerada correta; 3. "Qual a causa mais comum para a baixa produção de leite materno?", sendo as respostas "baixa sucção ou baixa ordenha" consideradas corretas; 4 . "Qual a causa mais comum de ingurgitamento mamário?", sendo as respostas "baixa sucção ou baixa ordenha" consideradas corretas. O não acerto em pelo menos um item foi considerado conhecimento inadequado.

As habilidades dos profissionais de saúde no manejo da amamentação foram avaliadas por meio de duas perguntas, sendo considerado adequado quando o profissional obteve respostas condizentes em duas questões: 1. "Você poderia mostrar como orientaria uma mãe a amamentar?"; 2. "Você poderia mostrar como orientaria uma mãe a fazer a expressão manual do leite?"

Por fim, duas perguntas foram utilizadas para aferir as práticas profissionais no manejo da amamentação, sendo considerado adequado quando ocorria resposta afirmativa às duas questões: 1 . "Você demonstra ou ensina as mães como fazer com seus bebês em relação ao posicionamento e pega para amamentar?"; 2. "Você demonstra ou ensina as mães como fazer a expressão manual do leite?".

Os três desfechos, dicotomizados, foram utilizados nas análises bivariadas e multivariadas, sendo obtidas razões de prevalência ajustadas por meio de modelo de regressão de Poisson com variância robusta ${ }^{14}$. Foram consideradas na análise multivariada apenas as variáveis cuja associação com cada desfecho apresentou p-valor menor ou igual a $20 \%$ (p-valor $\leq 0,20)$. Apesar de no modelo final terem sido apresentadas todas as variáveis explicativas que mostraram associação com $p$-valor $\leq 0,20$ na análise bivariada, apenas as associações que apresentaram p-valor menor ou igual a $5 \%(\mathrm{p}$-valor $\leq 0,05)$ foram consideradas significativas.

\section{Resultados}

Foram entrevistados, ao todo, 215 profissionais de saúde nos quinze hospitais com mais de 1000 partos/ano no município do Rio de Janeiro. Trabalhavam em hospitais amigos da criança 104 profissionais $(48,4 \%)$. A maioria dos profissionais era do sexo feminino (90,2\%), sendo $28,8 \%$ médicos, $56,7 \%$ profissionais da área da enfermagem e $14,5 \%$ de outras categorias. Quanto ao tempo de trabalho, $56,7 \%$ dos profissionais de saúde entrevistados tinham de 6 meses a 9 anos e 43,3\% de 10 a 45 anos de trabalho (Tabela 1 ).

Do total de profissionais entrevistados, $87,0 \%$ referiram ter feito alguma capacitação, dos quais a grande maioria pelo treinamento da $\operatorname{IHAC}^{9} \mathrm{e}$ $3,7 \%$ mediante outros cursos, como o curso de Aconselhamento ${ }^{15}$ ou o curso da Iniciativa Unidade Básica Amiga da Amamentação ${ }^{16}$. Alguns profissionais $(13,0 \%)$ não tinham recebido qualquer capacitação, $21,4 \%$ tinham realizado um treinamento apenas teórico ou com carga horária inferior a 18 horas, e 65,6\% dos profissionais tinham uma capacitação adequada (Tabela 1).

Os conhecimentos mais difundidos entre os profissionais de saúde foram os relativos à oferta de outro alimento interferindo na amamentação $(95,3 \%)$ e à causa principal do ingurgitamento mamário (93,0\%), enquanto $75,8 \%$ conheciam a principal causa de dor no mamilo e $62,8 \%$ a causa mais comum para a baixa produção de leite materno. Menos da metade dos profissionais $(48,1 \%)$ responderam adequadamente às quatro questões que aferiram o conhecimento (Tabela 2).

Quanto às habilidades dos profissionais de saúde, $86,0 \%$ souberam demonstrar adequadamente o posicionamento do bebê para mamar e a 
Tabela 1. Número e proporção de profissionais de saúde segundo o perfil nos 15 hospitais do Sistema Único de Saúde com mais de 1.000 partos/ano no município do Rio de Janeiro, 2009.

\begin{tabular}{lrr}
\hline \multirow{2}{*}{$\begin{array}{c}\text { Características dos } \\
\text { profissionais de saúde }\end{array}$} & \multicolumn{2}{c}{ Total } \\
\cline { 2 - 3 } & \multicolumn{1}{c}{$\mathbf{N}$} & $\%$ \\
\hline Capacitação & & \\
Adequada ( $\geq 18$ horas teórico-práticas) & 141 & 65,6 \\
Inadequada (<18 horas teórico-práticas) & 46 & 21,4 \\
Não capacitado & 28 & 13,0 \\
Trabalha em Hospital Amigo da Criança & & \\
Sim & 104 & 48,4 \\
Não & 111 & 51,6 \\
Sexo & & \\
Feminino & 194 & 90,2 \\
Masculino & 21 & 9,8 \\
Tempo de trabalho & & \\
6 meses - 9 anos & 122 & 56,7 \\
$\geq 10$ anos & 93 & 43,3 \\
Função & & \\
Obstetra & 28 & 13,0 \\
Pediatra & 34 & 15,8 \\
Enfermeiro & 53 & 24,6 \\
Auxiliar ou técnico de enfermagem & 69 & 32,1 \\
Nutricionista & 15 & 7,0 \\
Assistente social & 7 & 3,3 \\
Fonoaudiólogo & 4 & 1,9 \\
Psicólogo & 3 & 1,4 \\
Terapeuta ocupacional / fisioterapeuta & 2 & 0,9 \\
& & \\
\hline
\end{tabular}

pega da região mamilo areolar e 62,1\% demonstraram a ordenha manual da mama adequadamente. Dispunham de habilidades adequadas tanto na pega/posicionamento do bebê quanto em ordenha manual, 58,9\% dos profissionais (Tabela 3).

Já em relação à prática profissional, declararam que orientavam as mães quanto ao posicionamento e pega $89,8 \%$ dos profissionais de saúde e $78,1 \%$ que ensinavam às mães a ordenha manual da mama. Do total de entrevistados, 74,9\% relataram uma prática adequada em relação a estas duas orientações (Tabela 3 ).

Na Tabela 4 pode ser observado que, na análise bivariada, tanto a capacitação adequada, quanto trabalhar em Hospital Amigo da Criança, se mostraram associados a todos os desfechos, enquanto ser profissional de saúde do sexo masculino associou-se a uma menor prevalência de prática adequada de orientação sobre o manejo da amamentação. Trabalhar há menos de dez anos no hospital mostrou-se associado a uma menor prevalência de conhecimento e a uma maior prática. Pertencer à categoria médica associou-se a menor prevalência de habilidades e práticas adequadas relativas ao manejo da amamentação, e pertencer a outras categorias profissionais, que não a de enfermagem, também se associou a menor prevalência de práticas $(\mathrm{p} \leq 0,20)$.

$\mathrm{Na}$ análise multivariada, a capacitação adequada mostrou associação estatisticamente significativa com os três desfechos. Os profissionais com tempo de trabalho inferior a 10 anos apresentaram conhecimentos menos adequados, mas relataram uma maior prática de orientação às gestantes e/ou mães sobre o manejo da amamentação. Profissionais de enfermagem relataram uma maior prática de orientação sobre o manejo da amamentação do que profissionais da categoria médica ou outras (Tabela 5).

\section{Discussão}

No presente estudo, a capacitação adequada mostrou uma associação direta significativa com o conhecimento, as habilidades e as práticas profissionais em aleitamento materno, fundamentais na assistência às gestantes, mães e bebês. A capacitação de profissionais de saúde tem sido um fator fundamental para a melhoria dos conhecimentos ${ }^{17-21}$, habilidades ${ }^{18,22}$ e práticas profissionais e hospitalares ${ }^{19,21,23}$, corroborando o preconizado pelo passo 2 da IHAC: capacitar toda a equipe de cuidados de saúde para a implementação de normas e rotinas favoráveis à amamentação ${ }^{9}$. A Política Nacional de Educação Permanente em Saúde (Portaria GM/MS nº1996, 20 de agosto de 2007) também corrobora a necessidade de transformar as práticas institucionais através da formação e do desenvolvimento dos profissionais de saúde, compreendendo o espaço de trabalho como um espaço de aprendizado e aprimoramento constantes.

Ser um profissional atuante em Hospital Amigo da Criança não mostrou associação significativa com o conhecimento, as habilidades e as práticas profissionais após o ajuste pelas demais variáveis. No Rio de Janeiro, as equipes de todos as maternidades do SUS estão sendo capacitadas na IHAC e, apesar de nem todos os hospitais cumprirem o conjunto dos passos estipulados, mesmo os não credenciados cumprem entre 3 e 9 passos $^{10}$. Este envolvimento de todos os hospitais do SUS com a IHAC pode ser uma possível fonte de explicação para a ausência de associação encontrada na análise multivariada entre o creden- 
Tabela 2. Número e percentual de profissionais que responderam corretamente às questões relativas ao conhecimento profissional nos 15 hospitais do Sistema Único de Saúde com mais de 1.000 partos/ano no município do Rio de Janeiro, 2009.

\begin{tabular}{|c|c|c|c|c|c|c|c|c|c|c|}
\hline \multirow{4}{*}{ Características } & \multicolumn{10}{|c|}{ Conhecimentos } \\
\hline & \multirow{2}{*}{\multicolumn{2}{|c|}{$\begin{array}{c}\begin{array}{c}\text { Oferta de } \\
\text { outro alimento } \\
\text { interfere na } \\
\text { amamentação }\end{array} \\
\text { Sim }\end{array}$}} & \multirow{2}{*}{\multicolumn{2}{|c|}{$\begin{array}{c}\begin{array}{c}\text { Principal } \\
\text { causa de dor } \\
\text { no mamilo }\end{array} \\
\text { Má pega } \\
\end{array}$}} & \multirow{2}{*}{\multicolumn{2}{|c|}{$\begin{array}{c}\begin{array}{c}\text { Causa mais } \\
\text { comum de } \\
\text { baixa produção } \\
\text { de leite materno }\end{array} \\
\begin{array}{c}\text { Sucção ou } \\
\text { ordenha baixa }\end{array} \\
\end{array}$}} & \multirow{2}{*}{\multicolumn{2}{|c|}{$\begin{array}{c}\begin{array}{c}\text { Causa mais } \\
\text { comum do } \\
\text { ingurgitamento } \\
\text { mamário }\end{array} \\
\begin{array}{c}\text { Sucção ou } \\
\text { ordenha baixa }\end{array} \\
\end{array}$}} & \multirow{2}{*}{\multicolumn{2}{|c|}{$\begin{array}{c}\text { Total de } \\
\text { profissionais } \\
\text { com acerto nas } \\
4 \text { questões }\end{array}$}} \\
\hline & & & & & & & & & & \\
\hline & $\mathbf{n}$ & $\%$ & $\mathbf{n}$ & $\%$ & $\mathbf{n}$ & $\%$ & $\mathbf{n}$ & $\%$ & $\mathbf{n}$ & $\%$ \\
\hline \multicolumn{11}{|l|}{ Capacitação } \\
\hline Adequado & 140 & 99,3 & 111 & 78,7 & 97 & 68,8 & 136 & 96,5 & 79/141 & 56,0 \\
\hline Inadequado / não capacitado & 64 & 86,5 & 52 & 70,3 & 38 & 51,4 & 64 & 86,5 & $24 / 73$ & 32,9 \\
\hline \multicolumn{11}{|l|}{$\begin{array}{l}\text { Trabalhar em Hospital Amigo } \\
\text { da Criança }\end{array}$} \\
\hline $\operatorname{Sim}$ & 102 & 98,1 & 79 & 76,0 & 70 & 67,3 & 101 & 97,1 & $56 / 104$ & 53,8 \\
\hline Não & 102 & 92,7 & 84 & 75,7 & 65 & 58,6 & 99 & 89,2 & $47 / 110$ & 42,7 \\
\hline \multicolumn{11}{|l|}{ Sexo } \\
\hline Feminino & 184 & 95,3 & 150 & 77,3 & 120 & 61,9 & 181 & 93,3 & $93 / 193$ & 48,2 \\
\hline Masculino & 20 & 95,2 & 13 & 61,9 & 15 & 71,4 & 19 & 90,5 & $10 / 21$ & 47,6 \\
\hline \multicolumn{11}{|l|}{ Tempo de trabalho } \\
\hline 6 meses -9 anos & 116 & 95,9 & 88 & 72,1 & 69 & 56,6 & 112 & 91,8 & $50 / 121$ & 41,3 \\
\hline$\geq 10$ anos & 88 & 94,6 & 75 & 80,6 & 66 & 71,0 & 88 & 94,6 & $53 / 93$ & 57,0 \\
\hline \multicolumn{11}{|l|}{ Categoria profissional } \\
\hline Médica & 58 & 95,1 & 43 & 69,4 & 47 & 75,8 & 59 & 95,2 & $33 / 61$ & 54,1 \\
\hline Enfermagem & 118 & 96,7 & 98 & 80,3 & 72 & 59,0 & 112 & 91,8 & $57 / 122$ & 46,7 \\
\hline Outros & 28 & 90,3 & 22 & 71,0 & 16 & 51,6 & 29 & 93,5 & $13 / 31$ & 41,9 \\
\hline Total & 204 & 95,3 & 163 & 75,8 & 135 & 62,8 & 200 & 93,0 & $103 / 214$ & 48,1 \\
\hline
\end{tabular}

ciamento na IHAC e os desfechos investigados.

Um maior tempo de trabalho foi um fator que contribuiu para o conhecimento em aleitamento materno, mas se associou a um menor relato de prática profissional de orientação à clientela sobre o seu manejo. Não foram encontrados estudos que investigassem a associação entre tempo de trabalho e conhecimentos e práticas profissionais, para fins de comparação com os resultados do presente estudo. Um maior tempo de trabalho pode representar uma possibilidade maior de exposição não só a capacitações, mas à troca de conhecimentos durante o cotidiano profissional. É cabível também supor que profissionais com maior tempo de trabalho apresentem práticas mais deficientes de orientação, quer pelo acúmulo de funções que vão assumindo, quer pela rotina de trabalho que pode ser desgastante e desestimular práticas que envolvam relações interpessoais ${ }^{15}$. Outra explicação plausível seria o viés de informação, profissionais mais novos podem se sentir menos seguros no local de tra- balho e mais propensos a relatar uma prática de orientação às gestantes e mães do que profissionais mais antigos.

A categoria profissional apresentou associação significativa com as práticas na análise multivariada, favorecendo enfermeiros. Vários estudos de intervenção que investigaram o efeito da capacitação de profissionais de saúde em aleitamento materno a nível hospitalar se concentraram na área de enfermagem ${ }^{17,19,20,23}$, ou apenas alcançaram capacitar esta categoria profissional, por falta de adesão da categoria médica ${ }^{24}$. Estes achados sugerem que a categoria de enfermagem é mais sensível e disponível à participação em capacitações sobre aleitamento materno, podendo também o ser para orientar mulheres gestantes e mães no manejo da amamentação ${ }^{25}$.

No entanto, deve-se salientar a importância da capacitação e do envolvimento de todas as categorias profissionais na orientação às gestantes e mães sobre os benefícios e o manejo do aleitamento materno para que a equipe tenha um 
Tabela 3. Número e percentual de profissionais que responderam corretamente às questões relativas às habilidades e práticas profissionais nos 15 hospitais do Sistema Único de Saúde com mais de 1.000 partos/ano no município do Rio de Janeiro, 2009.

\begin{tabular}{|c|c|c|c|c|c|c|}
\hline \multirow{4}{*}{ Características } & \multicolumn{6}{|c|}{ Habilidades } \\
\hline & \multirow{2}{*}{\multicolumn{2}{|c|}{$\begin{array}{c}\text { Posicionamento } \\
\text { e Pega } \\
\begin{array}{c}\text { Demonstração } \\
\text { adequada }\end{array}\end{array}$}} & \multirow{2}{*}{\multicolumn{2}{|c|}{$\begin{array}{c}\text { Ordenha manual } \\
\text { da mama } \\
\begin{array}{c}\text { Demonstração } \\
\text { adequada }\end{array}\end{array}$}} & \multirow{2}{*}{\multicolumn{2}{|c|}{$\begin{array}{c}\text { Total de profissionais } \\
\text { com acerto nas } 2 \\
\text { questões }\end{array}$}} \\
\hline & & & & & & \\
\hline & $\mathbf{n}$ & $\%$ & $\mathbf{n}$ & $\%$ & $\mathbf{n}$ & $\%$ \\
\hline \multicolumn{7}{|l|}{ Capacitação } \\
\hline Adequado & 125 & 88,7 & 101 & 71,6 & $95 / 141$ & 67,4 \\
\hline Inadequado / não capacitado & 60 & 81,1 & 32 & 43,8 & $31 / 73$ & 42,5 \\
\hline \multicolumn{7}{|c|}{ Trabalhar em Hospital Amigo da Criança } \\
\hline $\operatorname{Sim}$ & 89 & 85,6 & 73 & 70,2 & $67 / 104$ & 64,4 \\
\hline Não & 96 & 86,5 & 60 & 54,5 & $59 / 111$ & 53,6 \\
\hline \multicolumn{7}{|l|}{ Sexo } \\
\hline Feminino & 166 & 85,6 & 124 & 63,9 & $117 / 194$ & 60,3 \\
\hline Masculino & 19 & 90,5 & 9 & 45,0 & $9 / 20$ & 45,0 \\
\hline \multicolumn{7}{|l|}{ Tempo de trabalho } \\
\hline 6 meses -9 anos & 112 & 91,8 & 78 & 64,5 & $75 / 121$ & 62,0 \\
\hline$\geq 10$ anos & 73 & 78,5 & 55 & 59,1 & $51 / 93$ & 54,8 \\
\hline \multicolumn{7}{|l|}{ Categoria profissional } \\
\hline Médica & 53 & 85,5 & 31 & 50,8 & $29 / 61$ & 47,5 \\
\hline Enfermagem & 106 & 86,9 & 84 & 68,9 & $79 / 122$ & 64,8 \\
\hline Outros & 26 & 83,9 & 18 & 58,1 & $18 / 31$ & 58,1 \\
\hline Total & 185 & 86,0 & 133 & 62,1 & $126 / 214$ & 58,9 \\
\hline \multirow{4}{*}{ Características } & \multicolumn{6}{|c|}{ Práticas } \\
\hline & \multirow{2}{*}{\multicolumn{2}{|c|}{$\begin{array}{c}\text { Posicionamento } \\
\text { e Pega } \\
\text { Demonstra ou } \\
\text { ensina às mães } \\
\end{array}$}} & \multirow{2}{*}{\multicolumn{2}{|c|}{$\begin{array}{c}\text { Ordenha manual } \\
\text { da mama }\end{array}$}} & \multirow{2}{*}{\multicolumn{2}{|c|}{$\begin{array}{c}\text { Total de profissionais } \\
\text { com acerto nas } 2 \\
\text { questões }\end{array}$}} \\
\hline & & & & & & \\
\hline & $\mathbf{n}$ & $\%$ & $\mathbf{n}$ & $\%$ & $\mathbf{n}$ & $\%$ \\
\hline \multicolumn{7}{|l|}{ Capacitação } \\
\hline Adequado & 133 & 94,3 & 120 & 85,1 & $116 / 141$ & 82,3 \\
\hline Inadequado / não capacitado & 60 & 81,1 & 48 & 64,9 & $45 / 74$ & 60,8 \\
\hline \multicolumn{7}{|c|}{ Trabalhar em Hospital Amigo da Criança } \\
\hline Sim & 96 & 92,3 & 87 & 83,7 & $84 / 104$ & 80,8 \\
\hline Não & 97 & 87,4 & 81 & 73,0 & $77 / 111$ & 69,4 \\
\hline \multicolumn{7}{|l|}{ Sexo } \\
\hline Feminino & 176 & 90,7 & 155 & 79,9 & $149 / 194$ & 76,8 \\
\hline Masculino & 17 & 81,0 & 13 & 61,9 & $12 / 21$ & 57,1 \\
\hline \multicolumn{7}{|l|}{ Tempo de trabalho } \\
\hline 6 meses -9 anos & 115 & 94,3 & 99 & 81,1 & $97 / 122$ & 79,5 \\
\hline$\geq 10$ anos & 78 & 83,9 & 69 & 74,2 & $64 / 93$ & 68,8 \\
\hline \multicolumn{7}{|l|}{ Categoria profissional } \\
\hline Médica & 55 & 88,7 & 43 & 69,4 & $40 / 62$ & 64,5 \\
\hline Enfermagem & 114 & 93,4 & 107 & 87,7 & $104 / 122$ & 85,2 \\
\hline Outros & 24 & 77,4 & 18 & 58,1 & $17 / 31$ & 54,8 \\
\hline Total & 193 & 89,8 & 168 & 78,1 & $161 / 215$ & 74,9 \\
\hline
\end{tabular}

discurso homogêneo, pois orientações discordantes são menos efetivas em estender a duração da amamentação, por trazerem insegurança à clientela ${ }^{26}$. Taddei et al. ${ }^{27}$ sinalizaram que as mudanças significativas nas práticas hospitalares foram mais fortemente relacionadas ao compor- 
Tabela 4. Razões de Prevalência brutas $\left(\mathrm{RP}_{\mathrm{b}}\right)$ dos conhecimentos, habilidades e práticas adequadas segundo capacitação profissional e características dos profissionais de saúde. Município do Rio de Janeiro, 2009.

\begin{tabular}{|c|c|c|c|c|c|c|c|c|c|}
\hline \multirow{2}{*}{ Características } & \multicolumn{3}{|c|}{ Conhecimentos } & \multicolumn{3}{|c|}{ Habilidades } & \multicolumn{3}{|c|}{ Práticas } \\
\hline & $\mathbf{R P}_{\mathrm{b}}$ & IC95\% & p-valor & $\mathbf{R P}_{\mathrm{b}}$ & IC95\% & p-valor & $\mathbf{R P}_{\mathrm{b}}$ & IC95\% & p-valor \\
\hline \multicolumn{10}{|l|}{ Capacitação } \\
\hline Inadequado / não capacitado & 1 & & & 1 & & & 1 & & \\
\hline Adequado & 1,704 & $(1,19-2,44)$ & 0,004 & 1,587 & $(1,19-2,12)$ & 0,002 & 1,353 & $(1,11-1,65)$ & 0,003 \\
\hline \multicolumn{10}{|c|}{ Trabalhar em Hospital Amigo da Criança } \\
\hline Sim & 1 & & & 1 & & & 1 & & \\
\hline Não & 0,794 & $(0,60-1,05)$ & 0,106 & 0,833 & $(0,67-1,04)$ & 0,110 & 0,859 & $(0,74-1,00)$ & 0,055 \\
\hline \multicolumn{10}{|c|}{ 然 } \\
\hline Feminino & 1 & & & 1 & & & 1 & & \\
\hline Masculino & 0,988 & $(0,62-1,58)$ & 0,961 & 0,746 & $(0,45-1,23)$ & 0,249 & 0,744 & $(0,51-1,09)$ & 0,126 \\
\hline \multicolumn{10}{|l|}{ Tempo de trabalho } \\
\hline$\geq 10$ anos & 1 & & & 1 & & & 1 & & \\
\hline 6 meses -9 anos & 0,725 & $(0,55-0,96)$ & 0,023 & 1,130 & $(0,90-1,42)$ & 0,299 & 1,155 & $(0,98-1,36)$ & 0,084 \\
\hline \multicolumn{10}{|l|}{ Categoria profissional } \\
\hline Enfermagem & 1 & & & 1 & & & 1 & & \\
\hline Médica & 1,158 & $(0,86-1,56)$ & 0,336 & 0,734 & $(0,55-0,99)$ & 0,040 & 0,757 & $(0,62-0,92)$ & 0,006 \\
\hline Outros & 0,898 & $(0,57-1,42)$ & 0,642 & 0,897 & $(0,65-1,24)$ & 0,513 & 0,643 & $(0,46-0,89)$ & 0,008 \\
\hline
\end{tabular}

Tabela 5. Razões de Prevalência ajustadas $\left(\mathrm{RP}_{\mathrm{a}}\right)$ dos conhecimentos, habilidades e práticas adequadas segundo capacitação profissional e características dos profissionais de saúde. Município do Rio de Janeiro, 2009.

\begin{tabular}{|c|c|c|c|c|c|c|c|c|c|}
\hline \multirow{2}{*}{ Características } & \multicolumn{3}{|c|}{ Conhecimentos } & \multicolumn{3}{|c|}{ Habilidades } & \multicolumn{3}{|c|}{ Práticas } \\
\hline & $\mathbf{R P}_{\mathrm{a}}$ & IC95\% & p-valor & $\mathbf{R P}_{\mathrm{a}}$ & IC95\% & p-valor & $\mathbf{R P}_{\mathrm{a}}$ & IC95\% & p-valor \\
\hline \multicolumn{10}{|l|}{ Capacitação } \\
\hline Inadequado / não capacitado & 1 & & & 1 & & & 1 & & \\
\hline Adequado & 1,575 & $(1,09-2,28)$ & 0,016 & 1,530 & $(1,13-2,06)$ & 0,005 & 1,312 & $(1,08-1,59)$ & 0,006 \\
\hline \multicolumn{10}{|c|}{ Trabalhar em Hospital Amigo da Criança } \\
\hline Sim & 1 & & & 1 & & & 1 & & \\
\hline Não & 0,838 & $(0,63-1,11)$ & 0,223 & 0,932 & $(0,74-1,17)$ & 0,541 & 0,968 & $(0,83-1,13)$ & 0,672 \\
\hline \multicolumn{10}{|l|}{ Sexo } \\
\hline Feminino & - & & & - & & & 1 & & \\
\hline Masculino & - & - & - & - & - & - & 0,770 & $(0,55-1,08)$ & 0,130 \\
\hline \multicolumn{10}{|l|}{ Tempo de trabalho } \\
\hline$\geq 10$ anos & 1 & & & - & & & 1 & & \\
\hline 6 meses -9 anos & 0,723 & $(0,55-0,95)$ & 0,021 & - & - & - & 1,183 & $(1,02-1,38)$ & 0,032 \\
\hline \multicolumn{10}{|l|}{ Categoria profissional } \\
\hline Enfermagem & & & & 1 & & & 1 & & \\
\hline Médica & - & & & 0,758 & $(0,57-1,01)$ & 0,060 & 0,808 & $(0,67-0,97)$ & 0,024 \\
\hline Outros & - & - & - & 0,947 & $(0,69-1,29)$ & 0,734 & 0,658 & $(0,49-0,89)$ & 0,007 \\
\hline
\end{tabular}

tamento dos profissionais do que às mudanças estruturais. Para que as mudanças nas práticas hospitalares tragam benefícios claros à clientela, uma equipe multidisciplinar deve ser considerada e valorizada, inclusive com a inclusão de staff administrativo, uma vez que essa categoria pro- 
pitais amigos da criança brasileiros credenciados entre 1992 e $2000^{29}$. No município do Rio de Janeiro, Oliveira et al. ${ }^{10}$ encontraram que o passo 2 estava sendo cumprido em 2009 por $71,4 \%$ dos hospitais reavaliados quanto à IHAC, e por apenas $12,5 \%$ dos não credenciados, revelando a lacuna ainda existente na capacitação adequada do staff destes estabelecimentos.

O presente estudo possui algumas limitações que devem ser destacadas. A adequação da capacitação e a prática profissional foram avaliadas por meio de informações prestadas pelos próprios profissionais durante a entrevista, o que pode implicar em viés de informação. O questionário não contemplou variáveis explicativas presentes em outras investigações, como a idade e a situação conjugal do profissional de saúde ${ }^{30}$, por não fazerem parte do escopo da pesquisa.

A Iniciativa Hospital Amigo da Criança, que tem tido um importante papel no aumento da prevalência da amamentação e do aleitamento materno exclusivo, está se expandindo no município do Rio de Janeiro, motivando os profissionais de saúde a atuarem na promoção, proteção e apoio ao aleitamento materno de forma ativa. O aumento na prática do aleitamento materno observado em diversas pesquisas é fruto da intensificação de ações de promoção da amamentação no país e na cidade nas últimas décadas ${ }^{31}$.

A partir do presente estudo conclui-se que uma capacitação adequada em aleitamento materno contribui para os conhecimentos, as habili- dades e as práticas profissionais, fundamentais à assistência a gestantes, mães e recém-natos.

Com vistas ao aprimoramento das práticas assistenciais de promoção, proteção e apoio à amamentação, recomenda-se a capacitação profissional continuada, dentro dos princípios da Educação Permanente em Saúde, não apenas voltada aos profissionais de saúde da área hospitalar, mas também aos que atuam na assistência primária. Recomenda-se também o monitoramento destas práticas, para que os patamares de qualidade alcançados ao longo do processo de credenciamento de estabelecimentos hospitalares na Iniciativa Hospital Amigo da Criança ${ }^{9}$ e de estabelecimentos de atenção primária na Iniciativa Unidade Básica Amiga da Amamentação ${ }^{16}$ não se deteriorem com o tempo. Neste sentido, vale ressaltar a recente proposta da OMS/UNICEF de automonitoramento anual, que permite um papel ativo das equipes hospitalares na manutenção das práticas preconizadas pela IHAC ${ }^{32}$.

Por fim, é preciso que pesquisas futuras investiguem variáveis ainda não exploradas em análises ajustadas, como o número de filhos dos profissionais de saúde, a amamentação pregressa, o grau de instrução, o turno de trabalho e o setor de atuação no hospital. Estudos que avaliem aspectos que podem influenciar uma capacitação adequada, sua periodicidade ideal e os fatores que favorecem e dificultam a adesão profissional a estas capacitações também são bem-vindos, tendo em vista a melhoria da qualidade da assistência ao público materno-infantil. 


\section{Colaboradores}

PC Jesus foi a responsável principal pela concepção do estudo, pela análise e interpretação dos dados e pela redação do artigo e da aprovação da versão a ser publicada. MIC Oliveira cuidou do desenho do estudo, da análise e interpretação dos dados, da redação do artigo e da aprovação da versão a ser publicada. JR Moraes participou da análise e interpretação dos dados, da revisão crítica do artigo e da aprovação da versão a ser publicada.

\section{Referências}

1. World Health Organization (WHO). The optimal duration of exclusive breastfeeding: results of a WHO systematic review. Geneva: WHO; 2001.

2. Boccolini CS, Boccolini PMM, Carvalho ML, Oliveira MIC. Padrões de aleitamento materno exclusivo e internação por diarréia entre 1999 e 2008 em capitais brasileiras. Cien Saude Colet 2012; 17(7):1857-1863.

3. Kramer MS, Chalmers B, Hodnett ED, Sevkovskaya Z, Dzikovich I, Shapiro S, Collet JP, Vanilovich I, Mezen I, Ducruet T, Shishko G, Zubovich V, Mknuik D, Gluchanina E, Dombrovskiy V, Ustinovitch A, Kot T, Bogdanovich N, Ovchinikova L, Helsing E; PROBIT Study Group (Promotion of Breastfeeding Intervention Trial). Promotion of Breastfeeding Intervention Trial (PROBIT): a randomized trial in the Republic of Belarus. JAMA 2001; 285(4):413-420.

4. Boccolini CS, Carvalho ML, Oliveira MIC, Boccolini PMM. Breastfeeding can prevent hospitalization for pneumonia among children under 1 year old. J Pediatr 2011; 87(5):399-404.

5. Thulier D, Mercer J. Variables associated with breastfeeding duration. J Obstet Gynecol Neonatal Nurs. 2009; 38(3):259-268

6. Abrahams SW, Labbok MH. Exploring the impact of the Baby-Friendly Hospital Initiative on trends in exclusive breastfeeding. Int Breastfeed J. 2009; 4:11.

7. Braun MLG, Giugliano ERJ, Soares MEM, Giugliani C, Oliveira AP, Danelon CMM. Evaluation of the impact of the Baby-Friendly Hospital Initiative on rates of breastfeeding. Am J Public Health 2003; 93(8):12771279.

8. Caldeira AP, Gonçalves E. Assessment of the impact of implementing the Baby-Friendly Hospital Initiative. J Pediatr. 2007; 83(2):127-132.

9. World Health Organization (WHO), United Nations Children's Fund (UNICEF). Baby-Friendly Hospital Initiative: Revised, Updated and Expanded for Integrated Care. Section 1: Background and Implementation. Geneva, New York: WHO, UNICEF, 2009.

10. Oliveira MIC, Hartz ZMA, Nascimento VC, Silva KS Avaliação da implantação da iniciativa hospital amigo da criança no Rio de Janeiro, Brasil. Rev. Bras. Saúde Matern. Infant. 2012; 12(3):281-295.

11. Bolfarine H, Bussab WO. Elementos da Amostragem. São Paulo: Blucher; 2005.

12. Brasil. Ministério da Saúde (MS). Módulo 5 - Avaliação e reavaliação externas, OMS/UNICEF. Seção 5.3 Orientações e instrumentos de reavaliação externa. Brasília: MS; 2009.

13. Fundo das Nações Unidas para a Infância (UNICEF Brasil). Iniciativa Hospital Amigo da Criança: revista atualizada e ampliada para o cuidado integral: módulo 2: fortalecendo e sustentando a iniciativa hospital amigo da criança: um curso para gestores. Brasília: Ministério da Saúde; 2009.

14. Barros AJD, Hirakata VN. Empirical comparison of models that directly estimate the prevalence ratio. BMC Med Res Methodol 2003 [acessado 2015 set 3]; 3:21. Disponível em: http://www.biomedcentral. com/1471-2288/3/21

15. Bueno LG, Teruya KM. The practice of breastfeeding counseling. J Pediatr (Rio J). 2004; 80(5 Supl.):S126-S130. 
16. Oliveira MIC, Camacho LAB, Tedstone AE. A method for the evaluation of primary care unit's practice in the promotion, protection, and support of breastfeeding: results from the State of Rio de Janeiro, Brazil. J Hum Lact. 2003; 19(4):365-373.

17. Hernández-Garduño AG, de la Rosa-Ruiz L. Breastfeeding training for the nursing staff at the General Hospital of Mexico. Salud Publica Mex. 2000; 42(2):112-117.

18. Moran VH, Bramwell R, Dykes F, Dinwoodie K. An evaluation of skills acquisition on the WHO/UNICEF Breastfeeding Management Course using the pre-validated Breastfeeding Support Skills Tool (BeSST). Midwifery 2000; 16(3):197-203

19. Owoaje ET, Oyemade A, Kolude OO. Previous BFHI training and nurses' knowledge, attitudes and practices regarding exclusive breastfeeding. Afr. Med Med Sci 2002; 31(2):137-140

20. Bernaix LW, Beaman ML, Schmidt CA, Harris JH, Miller LM. Success of an educational intervention on maternal/newborn nurses' breastfeeding knowledge and attitudes. JOGNN 2010; 39(6):658-666.

21. Zakarija-Grkovic I, Burmaz T. Effectiveness of the UNICEF/WHO 20-hour course in improving health professionals' knowledge, practices, and attitudes to breastfeeding: before/after study of 5 maternity facilities in Croatia. Croat Med J 2010; (51):396-405.

22. Rea MF, Venancio SI, Martines JC, Savage F. Counselling on breastfeeding: assessing knowledge and skills. Bull World Health Organ. 1999; 77 (6):492-498.

23. Martens PJ. Does breastfeeding education affect nursing staff beliefs, exclusive breastfeeding rates, and $\mathrm{Ba}$ by-Friendly Hospital Initiative compliance? The experience of a small, rural Canadian hospital. J Hum Lact 2000; 16(4):309-318

24. Coutinho SB, Lima M de C, Ashworth A, Lira PI. The impact of training based on the Baby-Friendly Hospital Initiative on breastfeeding practices in the Northeast of Brazil. J Pediatr 2005; 81(6):471-477.

25. Sydronio K, Souza IEO, Almeida JAG. Amamentação e enfermagem: análise descritiva e relevância da produção de pós-graduação. $R$ Enferm UERJ, 2006; 14(1):107-112.
26. Oliveira MIC, Camacho LAB, Tedstone AE. Extending breastfeeding duration through primary care: a systematic review of prenatal and postnatal interventions. J Hum Lact 2001; 17(4):326-343.

27. Taddei JAAC, Westphal MF, Venancio S, Bogus C, Souza S. Breastfeeding training for health professionals and resultant changes in breastfeeding duration. São Paulo Med J/ Rev Paul Med 2000; 118(6):185-191.

28. Khoury AJ, Hinton A, Mitra AK, Carothers C, Foretich C. Improving breastfeeding knowledge, attitudes, and practices of WIC Clinic Staff. Public Health Rep 2002; (117):453-462

29. Araújo MFM, Schmitz BAS. Doze anos de evolução da Iniciativa Hospital Amigo da Criança no Brasil. Rev Panam Salud Publica 2007; 22(2):91-99.

30. Davies-Adetugbo AA, Fabiyi AK, Ojoofeitimi EO, Adetugbo K. Breastfeeding training improves health worker performance in rural Nigeria. East Afr Med J 1997; 74(8):510-513

31. Castro IRR, Engstrom EM, Cardoso LO, Damião JJ, Rito RVFV, Gomes MASM. Tendência temporal da amamentação na cidade do Rio de Janeiro: 1996-2006. Rev Saude Publica 2009; 43(6):1021-1029.

32. Toma TS, Rea MF. La Iniciativa Hospital Amigo de La Niñez y la herramienta computarizada para su monitoreo y sustentabilidad. Rev Panam Salud Publica 2011; 30(5):505-506.

Artigo apresentado em 03/06/2015

Aprovado em 21/09/2015

Versão final apresentada em 23/09/2015 